\title{
Religion, histoire et société dans le monde grec antique
}

\section{Vinciane Pirenne-Delforge}

\section{(2) OpenEdition \\ 1 Journals}

\section{Édition électronique}

URL : https://journals.openedition.org/annuaire-cdf/15254

DOI : 10.4000/annuaire-cdf. 15254

ISBN : 978-2-7226-0572-5

ISSN : 2109-9227

\section{Éditeur}

Collège de France

Édition imprimée

Date de publication : 30 décembre 2020

Pagination : 227-239

ISBN : 978-2-7226-0516-9

ISSN : 0069-5580

\section{Référence électronique}

Vinciane Pirenne-Delforge, "Religion, histoire et société dans le monde grec antique », L'annuaire du Collège de France [En ligne], 118 | 2020, mis en ligne le 01 avril 2021, consulté le 22 août 2022. URL : http://journals.openedition.org/annuaire-cdf/15254; DOI : https://doi.org/10.4000/annuaire-cdf. 15254 


\title{
RELIGION, HISTOIRE ET SOCIÉTÉ DANS LE MONDE GREC ANTIQUE
}

\author{
Vinciane PIRENNE-DELFORGE \\ Professeure au Collège de France
}

Mots-clés : polythéisme, religion, sacrifice grec, Hérodote

La série de cours «Polythéisme grec, mode d'emploi » est disponible en audio et/ou en vidéo, sur le site internet du Collège de France (https://www.college-de-france.fr/ site/vinciane-pirenne-delforge/course-2017-2018.htm), ainsi que la leçon inaugurale "Religion, histoire et société dans le monde grec antique " (https://www.college-defrance.fr/site/vinciane-pirenne-delforge/inaugural-lecture-2017-2018.htm). La leçon inaugurale, Le Polythéisme grec comme objet d'histoire, est également publiée sous forme imprimée (Collège de France/Fayard, 2018) et numérique (Collège de France, 2019, https://books.openedition.org/cdf/6159).

\section{ENSEIGNEMENT}

COURS - POLYTHÉISME GREC, MODE D'EMPLOI

\section{Introduction}

À la suite de ma leçon inaugurale, qui posait le polythéisme grec comme objet d'histoire, le titre imagé de cette première série de cours évoque la nécessité de définir d'emblée certains fondements méthodologiques des enquêtes à venir. Or, parmi les problématiques amplement débattues pour appréhender la diversité du système religieux des Grecs, émerge rapidement celle de la tension entre le général et le particulier, entre l'unité et la diversité qui le caractérise et que traduisent quelques questions précises. Doit-on parler de religion grecque au singulier ou au pluriel ? Les figures divines se dissolvent-elles dans la variété des lieux où elles reçoivent un culte ou bien est-on en mesure de leur attribuer un profil spécifique partagé à plus large échelle ? Les actes sacrificiels ne sont-ils fondés que sur des pratiques strictement locales ou bien existe-t-il un arrière-plan commun à toutes les 
communautés grecques ? Après une réflexion préliminaire sur les notions mêmes de «religion» et de «polythéisme», les cours de cette année ont fait le point sur la manière dont ce questionnement permet d'aborder le fonctionnement de deux des composantes essentielles du polythéisme grec que sont la représentation des dieux et les démarches sacrificielles, en prenant comme fil rouge l'Enquête d'Hérodote.

\section{Religion et polythéisme}

Avant d'entrer dans le vif d'un sujet, il faut s'entendre sur les outils de travail que l'on mobilise. Or, à cet égard, les notions de religion et de polythéisme, qui sont au cœur de ma réflexion, offrent matière à controverse, à critique, voire à un rejet pur et simple (dans le cas de « religion») de la part de certains chercheurs. La question de la pertinence de ces termes en tant que concepts opératoires pour étudier les religions et les polythéismes n'est pas négligeable puisqu'un concept doit tendre vers un degré de généralisation justifiant qu'un discours puisse se dire scientifique.

Dans l'un et l'autre cas, c'est d'abord l'histoire des mots qui est convoquée ${ }^{1}$. La religio des Romains désigne en latin l'accomplissement scrupuleux des actions nécessaires à une bonne collaboration avec les dieux et, plus rarement, la manière adéquate de se comporter avec d'autres humains. Le registre d'application de la notion change profondément quand les Pères de l'Église s'emparent du terme à leurs propres fins. Ainsi, quand Tertullien écrit son Apologétique en 197 de notre ère, il l'investit pour désigner ce qu'il appelle nostra religio. Mais l'infléchissement ainsi opéré conduit à qualifier le terme de religio d'une manière inusitée. Il s'agit cette fois de la vera religio veri Dei, «la vraie religion du vrai Dieu » (XXIV, 1-2), ce qu'Augustin ne manquera pas de souligner encore deux siècles plus tard. La religio renvoie désormais au lien vertical, personnel, à un Dieu unique dont le message fonde toute autorité. En conséquence se dessine une distinction - au moins théorique et polémique - entre la sphère religieuse et la sphère sociale, voire la sphère politique (avec de nombreuses nuances), que les polythéismes antiques n'ont pas faite. De plus, le registre de la vérité et de l'erreur est étranger aux représentations de ces systèmes religieux. Faudrait-il dès lors cantonner le terme religion à la seule étude du christianisme qui l'a profondément marqué de son empreinte ? Pas forcément, car les alternatives ne sont pas satisfaisantes. En outre, si l'on choisit de s'en tenir au seul vocabulaire utilisé par les communautés que l'on étudie, on se prive d'office de toute possibilité de généralisation et de comparaison. À condition de repenser et d'affiner sans cesse la définition que l'on peut donner du terme en fonction des contextes auxquels on l'applique, ce terme opératoire conserve sa pertinence et je parle dès lors de «religion grecque » en tant qu' «institution qui régit, selon des modèles culturels [en l'occurrence ceux du monde grec antique], les relations avec la sphère supra-humaine dont cette culture postule l'existence ${ }^{2} \gg$.

1. Dans une bibliographie pléthorique, j'épingle M. SACHOT, «Origine et trajectoire d'un mot : religion » (Revue de philosophie ancienne, vol. 2003/2, 2005 ; en ligne http://www.univlyon3.fr/servlet/com.univ.collaboratif.utils.LectureFichiergw?ID_FICHIER=1287570601062) et J.Z. SMITH, « Religion, religions, religieux », in : Magie de la comparaison et autres essais d'histoire des religions, Genève, Labor et Fides, 2014, p. 29-52 (original anglais de 1998).

2. Cette définition (légèrement modifiée) est empruntée à M.E. SPIRO, «La religion : problème de définition et d'explication » [1966], in R.E. BRADBURY, C.C. GEERTZ, 
Quant au polythéisme, l'adjectif polytheos est attesté dans une tragédie d'Eschyle au $\mathrm{V}^{\mathrm{e}}$ siècle avant notre ère, mais cet emploi reste isolé avant que Philon d'Alexandrie et les Pères de l'Église n'exploitent le champ sémantique de polytheos/polytheia pour désigner une caractéristique essentielle des systèmes religieux de la Méditerranée antique que côtoient judaïsme et christianisme ${ }^{3}$. C'est à Guillaume Budé que l'on doit d'avoir récupéré, au XVI ${ }^{\mathrm{e}}$ siècle, ces mots grecs en les translittérant en latin pour désigner l'idolâtrie et le paganisme antiques ${ }^{4}$, tandis que Jean Bodin forge le terme français de polythéisme pour stigmatiser les hérésies chrétiennes ${ }^{5}$. Ensuite, on assiste à un changement de paradigme, puisque le mot de polythéisme vient progressivement remplacer l'idolâtrie, en atténuant ainsi la charge négative que véhiculait ce terme hérité de la traduction grecque de la Bible. Enfin, la réflexion sur la religion et les religions menée par les différentes sciences humaines qui se mettent en place au $\mathrm{XIX}^{\mathrm{e}}$ siècle introduit le polythéisme parmi les étapes qu'aurait traversées l'histoire religieuse de l'humanité et le définit par contraste avec d'autres systèmes en -isme, comme l'animisme, le totémisme, le fétichisme, le monothéisme, etc. C'est dans la seconde moitié du $\mathrm{XX}^{\mathrm{e}}$ siècle que le terme de polythéisme devient un outil en soi - et pas seulement par contraste - pour désigner les systèmes religieux qui peuplent la sphère supra-humaine d'une pluralité d'entités. C'est dans cette perspective que je l'emploie volontiers, en alternance avec « religion».

\section{Hérodote, « historien des religions et du polythéisme » 6}

Plusieurs raisons président au choix de partir du texte d'Hérodote pour aborder les tensions entre le général et le particulier dans l'étude de la religion grecque antique : 1) l'abondance du matériau qu'il offre en matière de religion; 2) la posture spécifique de l'enquêteur à l'égard de ce matériau par rapport aux poètes qui l'ont précédé ; 3) la représentation de la religion grecque qui se révèle en creux dans les descriptions des coutumes étrangères évoquées dans les quatre premiers livres par un

M.E. SPIRO, V. TURNER et E.H. WinTER (dir.), Essais d'anthropologie religieuse, Paris, Gallimard, 1972, p. 109-152.

3. Eschyle, Suppliantes, 424 ; PhILON D'AleXANDrIe, De fuga et inventione, 114 ; De virtutibus, 214 ; EUSÈBE DE CÉSARÉE, Préparation évangélique, I, 6, 4 ; XIV, 16, 12. Sur les Pères de l'Église, voir F. MASSA, «Nommer et classer les religions aux $\mathrm{II}^{\mathrm{e}}-\mathrm{IV}^{\mathrm{e}}$ siècles : la taxinomie paganisme, judaïsme, christianisme », Revue de l'histoire des religions, $\mathrm{n}^{\circ} 234$, 2017, p. 689-715.

4. E.g. G. BuDÉ, De studio literarum recte et commode instituendo (1532), 1273-1277 (éd. de la Garanderie).

5. J. Bodin, De la démonomanie des sorciers (1580), I, 5.

6. Dans une bibliographie pléthorique, j'épinglerais: F. HARTOG, Le Miroir d'Hérodote. Essai sur la représentation de l'autre, Paris, Gallimard, 1980 [2001] ; R. THOMAS, Herodotus in Context: Ethnography, Science and the Art of Persuasion, Cambridge, Cambridge University Press, 2000 ; les articles des deux ouvrages collectifs suivants : E.J. BAKKER, I.J.F. DE JONG et H. VAN WeEs (dir.), Brill's Companion to Herodotus, Leyde, Brill, 2002 ; C. Dewald et J. MARINCOLA (dir.), The Cambridge Companion to Herodotus, Cambridge, Cambridge University Press, 2006 ; C. CALAME, « Hérodote, précurseur du comparatisme en histoire des religions ? Retour sur la dénomination et l'identification des dieux en régime polythéiste », in F. PRESCENDi et Y. Volokhine (dir.), Dans le laboratoire de l'historien des religions. Mélanges offerts à Ph. Borgeaud, Genève, Labor et Fides, 2011, p. 263-274. 
observateur « interne » au monde grec ; 4) le recours à la notion de « dieux grecs » dans toute une série d'épisodes des guerres médiques décrits dans les cinq derniers livres. C'est la notion de nomos («coutume», «tradition ») que l'on a d'abord explorée afin d'en comprendre la portée dans l'usage qu'en fait Hérodote, notamment en regard des débats de son temps sur l'opposition entre nomos et phusis. Cette dernière est entendue non comme "nature » (une catégorie qui n'est pas encore constituée à l'époque), mais comme la complexion de ce qui croît et se développe indépendamment de l'initiative humaine. Les nomoi sont donc des ensembles de comportements et d'usages aussi variés que les peuples qui les portent et, parmi ces usages, on trouve les relations avec la sphère supra-humaine dont quelques exemples tirés des premiers livres de l'Enquête ont été analysés.

Dès lors, du contraste dessiné en creux par Hérodote entre les coutumes des Perses, des Scythes, etc., et celle des Grecs, ressortent divers éléments qui permettent d'identifier les composantes des nomoi qui concernent les dieux. En outre, dans le cadre du livre II entièrement dédié à l'Égypte, l'enquêteur prend position sur les «affaires divines » et affirme qu'il n'en parlera pas, sauf en ce qui concerne les ounomata (II, 3 et 65). L'analyse des implications de cette restriction programmatique permet de mieux comprendre la place des dieux dans l'Enquête, notamment en regard de la poésie antérieure. Quant aux ounomata divins, la portée du terme s'éclaire dans le cadre de la véritable «histoire du polythéisme » que raconte Hérodote au début du livre II, depuis le temps reculé des Pélasges jusqu'aux Grecs parlant grec (chap. 50-52). Ce qu'il désigne du terme d'ounoma est moins le «nom » des dieux que leur « dénomination », c'est-à-dire le fait d'identifier, en leur attribuant une désignation spécifique, des profils divins au sein d'un ensemble de dieux qui, pour les Pélasges, étaient d'abord indifférenciés. Chaque peuple est susceptible de procéder ainsi et l'identification de tels «profils » divins peut ensuite être transmise d'un peuple à l'autre. Dans la logique diffusionniste mise en place par Hérodote, la grande ancienneté qu'il attribue aux Égyptiens les institue en généreux pourvoyeurs de «dénominations » divines. C'est ensuite aux poètes Homère et Hésiode qu'Hérodote attribue la complexification de la représentation des dieux propre aux Grecs (II, 53). La religion en général et la religion grecque en particulier, c'est-à-dire les nomoi qui concernent les dieux, auxquels viennent s'ajouter les créations poétiques, sont bien des institutions humaines, culturellement déterminées.

Mais qu'en est-il des « dieux grecs » ? S'agit-il d'une catégorie pertinente dans sa généralité ? Dans le cadre des cinq derniers livres de l'Enquête, quelques discours rapportés par Hérodote y font référence ainsi qu'aux «sanctuaires communs » qui font partie des composantes de la « grécité ». Que recouvrent ces catégories et dans quelle mesure nous permettent-elles de penser les dieux des Grecs? La communauté constituée par la fréquentation des sanctuaires communs - régionaux comme à Délos et/ou panhelléniques comme à Olympie - est une communauté rituelle transitoire, liée à un espace déterminé, et elle ne renvoie pas à une quelconque "identité religieuse » partagée (VIII, 144) ${ }^{7}$. Quant aux dieux qualifiés de «grecs » dans les

7. R. PARKER, Cleomenes on the Acropolis: An Inaugural Lecture Delivered before the University of Oxford on 12 May 1997, Oxford, Clarendon Press, 1998 ; I. POLINSKAYA, "Shared sanctuaries and the gods of others: On the meaning of "common" in Herodotus 8.144 », in R.M. Rosen et I. SLUITER (dir.), Valuing Others in Classical Antiquity, Leyde/ Boston, Brill, 2010, p. 43-70. 
discours de certains acteurs des guerres médiques, leur invocation sert à fédérer les forces grecques face à l'armée du roi des Perses (V, 49 ; 92-93 ; IX, 90). L'emphase rhétorique d'une telle qualification n'est pas à négliger, mais elle n'est pas seule en cause. En effet, dans l'emporion de Naucratis dont parle Hérodote et où il situe un sanctuaire appelé «Hellénion » (II, 178), l'archéologie a mis au jour des dédicaces « aux dieux des Grecs » qui attestent la portée rituelle d'une telle invocation ${ }^{8}$. Dans un environnement égyptien, l'effet de contraste est évident et justifie pour partie la présence d'un tel groupe générique, mais ce filon documentaire exceptionnel atteste aussi que «les dieux des Grecs » peuvent être une entité cultuelle destinataire de l'hommage des Grecs, quelles que soient leurs cités respectives.

Par ailleurs, l'Enquête elle-même montre que la dénomination des theoi, un processus qu'Hérodote fait remonter aux Pélasges instruits par les Égyptiens, s'adresse potentiellement à tous les Grecs. Qu'en est-il dès lors de la relation entre le nom d'un dieu présent dans cet imaginaire partagé par les Grecs et les surnoms - que l'on appelle des épiclèses - qui lui sont attribués à l'échelle locale ? Autrement dit, un dieu conserve-t-il, ou non, une unité derrière la multiplicité des cultes qu'il reçoit ? C'est vers une réponse positive que tend l'analyse des différents facteurs de multiplication des entités du monde supra-humain des Grecs. Que ce soit le groupe des « Douze dieux », les collectifs féminins comme les Charites ou les Muses, mais aussi chaque divinité dont le nom est au singulier, les Grecs les concevaient comme des puissances qui pouvaient se condenser ou se déployer selon les contextes et les attentes de ceux qui les honoraient. Les épiclèses ne faisaient pas « éclater » le dieu en autant de figures hétérogènes. Ainsi que l'affirmait Socrate mis en scène dans le Banquet de Xénophon (VIII, 9), «Zeus, qui paraît toujours le même, possède de nombreux surnoms » (cf. aussi Hdt., I, 44).

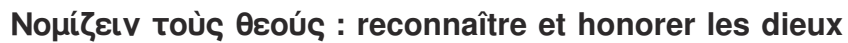

Une réflexion sur le polythéisme grec et sur le caractère opératoire de la notion de «religion» ne peut s'abstraire, aujourd'hui, d'un détour par la question de la

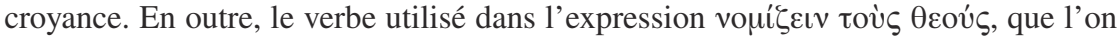
a parfois traduite par « croire aux dieux », s'inscrit dans le même champ sémantique que les nomoi dont l'importance est cruciale dans la conception de la religion que

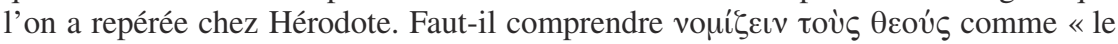
fait de croire aux dieux »? Et, si c'est le cas, quel lien l'observateur moderne peut-il nouer entre «tradition » et « croyance » ? La question est loin d'être anodine car elle engage les éventuels déterminismes culturels dont l'étude des religions a bien du mal à se débarrasser.

L'étude des différentes constructions du verbe vouí̧६ıv, d'une part, et, d'autre part, les différents emplois du verbe avec le complément $\theta \varepsilon$ ó $\varsigma$, avec ou sans article, au singulier ou au pluriel, atteste une appréhension des dieux qui fait écho, à l'échelle des cultes singuliers, à l'histoire générale du polythéisme telle que l'élabore

8. A. Johnston, « The Delta: From Gamma to Zeta », in A. Villing (dir.), Naukratis: Greek Diversity in Egypt. Studies on East Greek Pottery and Exchange in the Eastern Mediterranean, Londres, British Museum, 2006, p. 23-30 ; Id., « Ceramic inscriptions », in A. VILling et al. (dir.), Naukratis: Greeks in Egypt, Londres, British Museum, 2017 ; Id., «Inscriptions on Greek Vases », ibid. 
Hérodote. Pour lui, comme on l'a vu, des theoi indéterminés des Pélasges, on passe à l'identification des dieux par des noms (ounomata) et à la mise en place de nomoi spécifiques par les Grecs pour leur rendre hommage. Or, l'expression nomizein theon ou tous theous mêle étroitement deux dimensions : celle de la représentation - c'est l'ounoma de l'enquêteur, auquel les poètes ont ajouté d'autres éléments d'identification - et celle des cultes. La traduction, certes un peu complexe, de cette expression par « reconnaître et honorer comme dieu » ou « reconnaître et honorer les dieux », signifie cette dualité qui consiste à identifier un ou plusieurs dieu(x) et à le, ou les, inscrire officiellement dans les nomoi du groupe qui le, ou les, accueille. C'est tout cela qui est ainsi signifié et qui explique la difficulté de trouver un juste correspondant en français.

Est-il dès lors pertinent de conserver la catégorie du «croire » pour parler de religion grecque? Cette question a particulièrement agité les anthropologues confrontés, comme les historiens, à la difficulté d'exporter des notions en dehors des contextes spécifiques qui les ont vus naître. Needham, côté anglophone, et Pouillon, côté francophone, sont les références obligées de ces débats et des doutes sérieux qui se sont levés sur la «croyance» des communautés non chrétiennes ${ }^{9}$. Dans le cadre des études sur la religion grecque, la notion a fait un retour remarqué ces dernières années, dans un mouvement de réaction à l'identification des polythéismes antiques aux seuls rituels et au registre du « faire » ${ }^{10}$. Mais c'est à définir ce que l'on entend par « croyance» (ou «belief») qu'il faut s'attacher. Et de ce point de vue, la réflexion anthropologique est plus mûre que celles des antiquisants.

Ainsi, Roberte Hamayon, éminente spécialiste du chamanisme sibérien, a souligné avec finesse que l'une des difficultés de cette notion était l'usage que nous en faisons en désignant de la sorte à la fois ce que l'on croit et le fait même de croire. Dans l'usage que nous avons du verbe et du substantif associés (croire, croyance, croyant), la croyance est à la fois un contenu et l'adhésion à ce contenu (c'est d'ailleurs pour cela que l'on peut parler de « croyant $\left.{ }^{11} »\right)$. Nombre de cultures n'établissent pas un lien nécessaire entre ce que l'on croit (un contenu) et le fait de croire (l'adhésion à un contenu). L'objet de la croyance n'est pas exclusif, circonscrit et définitif : il est négociable, dans une attitude d'adhésion fluide, où l'objet de croyance peut être pluriel et flexible. Or, dans le cadre de ce qui est une forme de négociation avec des entités non humaines, les actes rituels sont essentiels, car ils sont autant de mises à l'épreuve de l'objet de croyance, inscrite dans une tradition. C'est ce que R. Hamayon appelle la dynamique spéculative inhérente aux cultures, où sont dissociés l'attitude croyante et l'objet de la croyance. Ce constat m'intéresse au plus haut point, car il rapproche le nomizein tous theous des Grecs des cultures de Mongolie et de Sibérie étudiées par l'anthropologue.

9. R. Needham, Belief, Language, and Experience, Chicago, B. Blackwell, 1972 ; J. POUILlON, « Remarques sur le verbe "croire" », in M. IZARD et P. SMITH (dir.), La Fonction symbolique. Essai d'anthropologie, Paris, Gallimard, 1979, p. 43-51 [repris dans Le Cru et le Su (1993), p. 17-36].

10. Voir le dossier bibliographique établi par T. HARRISON, «Beyond the polis? New approaches to Greek religion », Journal of Hellenic Studies, vol. 135, 2015, p. 165-280.

11. R.N. HAMAYON, «L'anthropologie et la dualité paradoxale du "croire" occidental », Revue du MAUSS, vol. 20, 2006, p. 427-448. 
Les Grecs croyaient que les dieux «étaient là » et qu'ils étaient partie prenante de la vie des hommes, ils croyaient qu'il était bénéfique de les honorer et de chercher le bon interlocuteur dans telle ou telle situation. Il s'agit de « croire que », c'est-à-dire la dimension cognitive du «croire » qui s'approche du registre du savoir, tout en laissant la porte ouverte au doute. Et le doute enclenche la dynamique spéculative qui passe à la fois par des représentations multiples - et qui peuvent nous paraître contradictoires - et des rituels qui servent de pierre de touche à la relation. Les rituels sont autant d'expérimentations de la validité et de l'efficacité de cette relation. C'est tout cela que charrie avec elle l'expression nomizein tous theous, « reconnaître et honorer les dieux». Et la traduire par «croire aux dieux» en aplatit considérablement le sens, tout en ouvrant la porte à de lourdes erreurs de perspective en raison du lien que nous opérons, dans nos cultures modernes, entre le fait de croire et l'objet de la croyance.

Dès lors, contrairement au terme de «religion» que j'ai d'emblée proposé de conserver, il me semble que l'on n'a aucun intérêt à conserver le registre du « croire en », du «croire à » ou, pire encore, le terme de «croyant» pour identifier la démarche des Grecs ou des Romains dans leurs relations avec la sphère suprahumaine. Je n'en ramène pas pour autant ces religions antiques au ritualisme froid auquel on les a trop longtemps confinés en leur déniant toute démarche de « foi » au sens de la confiance envers un interlocuteur supra-humain. Au contraire, c'est la richesse d'une relation différente au divin que je souhaite ainsi mettre en évidence, comme la lecture des auteurs anciens l'atteste. Nomizein tous theous signifie à la fois le fait de reconnaître les dieux (c'est-à-dire de les identifier, de les nommer, de se les représenter sous des figures diverses) et le fait de leur rendre les hommages qui leur sont dus. Les deux registres sont indissociables, mais cela ne fait pas pour autant des Grecs des « croyants ».

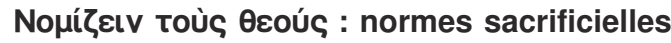

Reconnaître et honorer les dieux implique une démarche cognitive et des actes à poser. Dans le registre des représentations, l'unité et la diversité du système religieux des Grecs s'inscrit dans la tension entre les théonymes partagés, voire le groupe des « dieux des Grecs", et les titres cultuels localement attestés. Sur le plan des démarches rituelles, une même tension entre unité et diversité se joue surtout en matière sacrificielle. La confrontation des textes littéraires entre eux - essentiellement l'épopée homérique et les comédies d'Aristophane - atteste qu'il existe quelque chose comme une « trame sacrificielle », à savoir une structure minimale associée au mot de «sacrifice » qui, en grec classique, est la thusia. Cette trame est générale, d'un point de vue rituel, comme le nom de Zeus renvoie à une généralité de Zeus au-delà de ses manifestations singulières. Aux textes que je viens d'évoquer, on ajoutera un passage d'Hérodote - qui reste notre fil rouge - généralement peu exploité dans ce contexte. Il s'agit de la présentation du « sacrifice en usage chez les Perses » (I, 132) où l'enquêteur fait se succéder constats négatifs («ils ne dressent pas d'autel, ils n'allument pas de feu », etc.) et constats positifs («il conduit la bête dans un lieu pur, il invoque le dieu », etc.). Les propositions négatives offrent l'indice le plus sûr d'une comparaison différentielle avec les rituels grecs que l'on peut dès lors reconstituer «en creux». En outre, comme le point de vue d'Hérodote est globalement "grec », les éléments qu'il retient ont toute chance de se situer à un degré de généralité qui permet de transcender les particularités locales. 
Quand on rassemble les éléments à l'intersection du témoignage de l'épopée homérique, d'Hérodote et des comédies d'Aristophane, on voit se dessiner un rituel accompagné de musique où l'agent cultuel est facultatif, où l'autel enflammé est central et où les libations ponctuent les séquences à l'autel ; l'animal mis à mort est découpé en trois parts, la part du dieu (qui brûle sur l'autel, mais pourra aussi s'accompagner, hors épopée homérique, d'une déposition sur table), les viscères nobles auxquels les participants goûtent, le reste des viandes à consommer sur place ou non. Cette structure semble bien être une «manière grecque de sacrifier » et une norme rituelle épigraphique, publiée en 2015, a permis de trouver, pour la première

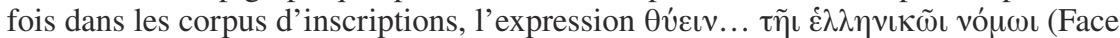
$\mathrm{B}$, lignes 34-35), à savoir « sacrifier selon la coutume grecque »-c'est bien le nomos qui apparaît à nouveau ici ${ }^{12}$. La nécessité d'une telle précision, inusitée dans les inscriptions prescriptives de ce type, est dictée par l'identité de l'association implicitement associée à ce culte : elle devait être composée essentiellement de dévots originaires du Proche-Orient (de Syrie ?), auxquels étaient susceptibles de se joindre des Grecs du cru, à savoir le cœur de la Thessalie. La mention explicite de la part brûlée à l'autel pour la déesse (accompagnée d'une déposition sur table) et des viscères à cuire (même si le mode de cuisson est inhabituel) sous l'appellation de hiera, de «parts sacrées » tend à confirmer la structure en trois temps (le reste des viandes étant induit) du sacrifice «à la grecque».

Une comparaison avec le traitement des viscères dans d'autres inscriptions émises par des cités ou des groupements grecs montre à la fois l'importance de ces éléments dans la «trame», mais aussi les nombreuses variations que les déterminations locales peuvent induire dans la manipulation des carcasses animales. En recourant à une métaphore commode, même si toute image a ses limites, on peut dire que la trame sacrificielle est comme une langue partagée par tous, tandis que les motifs particuliers qui viennent s'y greffer sont de l'ordre de la parole singulière. On souligne ainsi la part de contrainte du système, mais aussi sa part de liberté. Quant à la communication que permettent tant la langue que la parole, elle peut s'appliquer aussi au rituel sacrificiel qui l'autorise tant sur le plan vertical (la relation aux dieux) que sur le plan horizontal (la détermination d'une hiérarchie et de statuts à l'intérieur même de la communauté au nom de laquelle le sacrifice est offert).

C'est à préciser cette double communication sacrificielle que s'est aussi attachée la leçon sur les Tritopatores, ces « ancêtres du troisième degré » qui sont honorés dans divers endroits du monde grec et permettent aussi de considérer la tension entre le général et le particulier ${ }^{13}$.

12. J.-C. Decourt et A. Tziaphalias, «Un règlement religieux de la région de Larissa : cultes grecs et "orientaux" », Kernos, vol. 28, 2015, p. 13-51 ; J.-M. CARBON, « The Festival of the Aloulaia, and the Association of Alouliastiai. Notes concerning the new inscription from Larisa/Marmarini », Kernos, vol. 29, 2016, p. 185-208 ; R. PARKER, « The Nameless Goddess of Marmarini », Zeitschrift für Papyrologie und Epigraphik, vol. 199, 2016, p. 58-59 ; R. PARKER et S. SCULliON, «The Mysteries of the Goddess of Marmarini », Kernos, vol. 29, 2016, p. 209-266 ; R. BouCHON et J.-C. DECOURT, « Le règlement religieux de Marmarini (Thessalie) : nouvelles lectures, nouvelles interprétations », Kernos, vol. 30, 2017, p. 159-186.

13. Cette étude est désormais publiée : V. Pirenne-Delforge, «Pure and impure ancestors at Selinous: A note on Greek theology », in K. BIELAWSKI (dir.), Animal Sacrifice in Ancient Greece. Proceedings of the First International Workshop in Kraków (12-14/11/2015), Varsovie, Global Scientific Platform, 2017, p. 67-86. 


\section{Remarques conclusives}

Ces considérations finales ont permis de revenir sur la dimension topique du polythéisme grec, à savoir son ancrage dans un territoire. C'est à nouveau Hérodote qui a servi de guide en cette occasion conclusive. Au livre V (72), il décrit le roi de Sparte Cléomène qui, à la fin du $\mathrm{VI}^{\mathrm{e}}$ siècle avant notre ère, serait monté sur l'acropole d'Athènes, où s'élevait l'antique temple d'Athéna Polias, déesse tutélaire de la cité. Il aurait franchi la porte du temple et la prêtresse du lieu lui aurait intimé l'ordre de faire demi-tour au prétexte qu' « il n' [était] pas religieusement permis aux Doriens de se présenter là ». À quoi Cléomène aurait répondu qu'il n'était pas Dorien mais Achéen. Une telle scène, dont l'historicité est loin d'être assurée, est à rapprocher d'une autre occasion où le même Cléomène, voulant cette fois se rendre maître de la cité d'Argos, se rend dans le prestigieux sanctuaire d'Héra - déesse tutélaire de la cité elle aussi - à l'extérieur du centre urbain, pour y sacrifier lui-même à l'autel. Le prêtre surgit alors et lui déclare qu' « il n' [est] pas permis à un étranger de sacrifier en ce lieu ». Passant outre, le roi laisse maltraiter le prêtre par ses serviteurs et procède lui-même au sacrifice, tandis qu'une flamme peu engageante s'échappe de la poitrine de la statue (VI, 81-82).

L'économie narrative des deux épisodes n'est pas tout à fait la même, mais l'une et l'autre scène jouent sur des prescriptions sacrificielles bien connues du public et du lectorat d'Hérodote : l'interdiction totale parfois faite à un étranger, un xenos, d'accéder à un sanctuaire, d'une part, et, d'autre part, l'interdiction faite à un étranger de sacrifier lui-même dans une cité autre que la sienne. En effet, l'expression

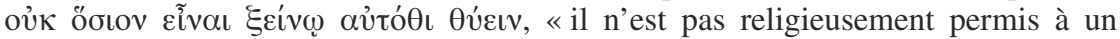
étranger de sacrifier ici », n'exclut pas forcément que l'étranger puisse en passer par un intermédiaire local pour procéder au sacrifice, voire par le prêtre lui-même, ainsi que l'attestent nombre d'inscriptions prescriptives. Car le xenos, c'est l'étranger avec lequel il est possible d'entrer en relation dans un partage de références communes, ne fût-ce que la langue parlée et un arrière-plan culturel partagé. Le substantif xenia ne désigne pas «l'étrangeté », mais bien «l'hospitalité », et la traduction de xenos par « étranger » n'est pas entièrement satisfaisante, surtout en un temps, le nôtre, où le terme s'est chargé de tout le poids d'une altérité distante. Le xenos n'avait pas accès aux rouages politiques d'une cité dont il n'était pas citoyen et les cités prévoyaient des mécanismes d'intermédiation. Mais l'accès aux institutions religieuses n'allait pas non plus de soi et, avant d'en arriver parfois à un interdit pur et simple comme les inscriptions en témoignent parfois, il existait un éventail de mesures permettant à ce type de visiteur d'accéder aux différents sanctuaires et aux feux sacrificiels de la cité qui l'accueillait. L'Athéna de Sparte (car elle trônait sur l'acropole de la cité de Cléomène) et l'Athéna d'Athènes n'étaient pas forcément des divinités différentes dans l'imaginaire du roi spartiate montant sur l'acropole de la cité athénienne. Mais les sanctuaires respectifs de ces déesses étaient locaux, ainsi que les règles qui en régissaient le culte. C'est bien dans la tension entre le général et le particulier que se joue, dans le monde grec, le nomizein tous theous.

Que signifie, dès lors, dans ce contexte, le fait que Cléomène se revendique achéen ? Dans le monde de Cléomène et celui d'Hérodote, être achéen signifie que l'on vient de la région située au nord du Péloponnèse, ce qui ne fait guère sens pour justifier la réplique du roi. En revanche, sous la plume du plus homérique des historiens, l'épisode prend une autre tournure. En effet, dans la tradition épique, et tout particulièrement dans l'Iliade, c'est ainsi que le poète désigne majoritairement 
les Grecs qui ont mis le siège sous les murailles de Troie. Ce sont les Achaioi. Ils peuvent aussi être qualifiés de Danaoi, mais ils sont près de cinq fois plus souvent appelés Achéens que Danaens. C'est dans cette référence aux guerriers grecs qui ont fait tomber Troie que se situe tout le sel de la réplique qu'Hérodote place dans la bouche du roi de Sparte. Ainsi, au chant VI de l'Iliade, la reine Hécube accompagnée de femmes troyennes et de la prêtresse Théanô s'en va déposer un linge éclatant sur les genoux de l'Athéna dont le temple orne l'acropole de la cité, pour la prier de soutenir l'armée troyenne. Mais la déesse n'agrée pas l'offrande, elle répond «non », nous dit le poète assumant sa position de surplomb par rapport aux protagonistes du récit, ces femmes pleines d'espoir de voir aboutir leur supplication (Il., VI, 297-311).

Dans la scène de Cléomène montant sur l'Acropole d'Athènes, c'est le « oui » d'Athéna à son entreprise de conquête que vient chercher le roi de Sparte, ou plutôt, pour conserver le strict parallèle avec le chant VI de l'Iliade, il vient quérir un «non » de la part de la déesse et son refus d'assurer la protection de sa propre cité, comme l'Athéna qui tenait l'acropole de Troie, la déesse qui a favorisé cet autre roi de Sparte qu'était Ménélas, au détriment des femmes et des enfants troyens. C'est un tel arrière-plan épique qui est convoqué par le statut d'Achéen qu'assume Cléomène face à la prêtresse athénienne, la Théanô de la cité d'Athènes. Mais cette Athéna-là n'est plus « hors sol » comme dans l'Iliade; à Athènes, elle est la protectrice de la cité et Cléomène sera chassé. Si j'ai raison de voir dans la revendication d'être un Achéen une référence à l'épopée, cette petite histoire atteste que, pour Hérodote et ses auditeurs/lecteurs, l'Athéna « hors sol» des traditions narratives pouvait entrer en résonance avec les cultes que les communautés locales lui rendaient sur leur acropole ou ailleurs en l'enracinant dans leur territoire. Et ce lien passait par le nom même de la divinité.

Les dieux grecs sont profondément topiques, locaux, au sens où c'est l'hommage rendu par les communautés qui fait d'eux les dieux qu'ils sont et non une quelconque révélation générique qui affirmerait leur existence et définirait les obligations de leurs «fidèles », de leurs «croyants ». Mais l'existence même d'un imaginaire partagé ne doit pas être négligée pour comprendre les choix qui étaient opérés à l'échelle locale par les communautés dans l'éventail de l'offre divine disponible. La relation avec les multiples puissances du monde supra-humain, qui faisaient communauté avec eux, était fondée sur une expérimentation continue. Et cette expérimentation mobilisait des représentations puisées à toutes les sources des savoirs sur les dieux, celles de l'expérience rituelle comme celles des traditions narratives et iconographiques, des plus sublimes aux plus modestes.

\section{Colloque - Autorité, NORMES et Rituels EN Grèce ANCIENNE. AutOUR DU PROJET COLLECTION OF GREEK RITUAL NORMS}

Certaines des inscriptions grecques que le hasard des fouilles nous a conservées touchent aux rituels qui étaient accomplis pour les dieux et ont une dimension prescriptive. Ces documents, longtemps qualifiés de «lois sacrées », émanent d'instances variées, déploient des prescriptions qui ne sont pas moins variées et offrent quantité d'informations sur les rituels accomplis par les cités, par les divers groupes qui les constituent, dont les maisonnées dans le cas de «fondations » familiales. C'est peu dire que ces textes nous livrent des données qui resteraient inaccessibles à l'historien de la religion grecque sans la moisson épigraphique 
régulière que livrent les fouilles systématiques ou les découvertes fortuites. Ces mal nommées « lois sacrées » sont au cœur du projet Collection of Greek Ritual Norms (CGRN) qui, outre le fait de les rebaptiser en «normes rituelles », en livre pour l'instant 222 exemplaires qui sont disponibles en ligne (http://cgrn.ulg.ac.be) et concernent les thèmes du sacrifice et de la purification. À moyen terme, le projet a l'ambition de constituer un recueil de quelque 500 textes et, outre la constitution de cet outil informatique, il entend donner l'occasion d'approfondir l'analyse de ces divers types de documents.

La rencontre de mai 2018 a réuni sur deux jours un groupe de chercheurs engagés, d'une manière ou d'une autre, dans l'étude de tels documents, anciennement et nouvellement découverts, en offrant soit une réflexion d'ensemble sur les implications du projet et sur la question des «normes rituelles » (E. Harris, R. Parker, A. et I. Petrovic, et E. Stavrianopoulou), soit une analyse de nouveaux documents (A.P. Matthaiou et D. Rousset), soit un regard neuf sur des documents déjà connus (S. Lambert et S. Caneva), soit enfin l'analyse de composantes essentielles du système religieux des Grecs telles que les normes rituelles permettent de l'aborder (S. Georgourdi et Z. Pitz sur le sacrifice, P. Brulé sur la notion de croyance). Une ouverture comparatiste a été offerte par la communication de J. Scheid sur le monde romain ${ }^{14}$.

\section{COURS À L'EXTÉRIEUR}

Université de Lausanne, avril 2018 :

1) «Normes rituelles grecques. Des "lois sacrées" qui ne sont ni des lois, ni sacrées »;

2) «Comment étudier les dieux grecs ? Le cas de la déesse Héra (avec Gabriella Pironti)».

\section{RECHERCHE}

\section{PROJETS DE RECHERCHE}

- Le général et le particulier dans le polythéisme grec à partir de la lecture d'Hérodote (cf. résumé des cours) ; réflexion sur la place des splanchna (viscères) dans le rituel sacrificiel grec.

- Participation au comité consultatif du projet ERC Mapping Ancient Polytheism dirigé par Corinne Bonnet à l'université de Toulouse Jean-Jaurès.

- Poursuite du projet CGRN (Collection of Greek Ritual Norms), en collaboration avec Jan-Mathieu Carbon, maître de conférence attaché à ma chaire ; le colloque évoqué ci-dessus s'inscrit directement dans la réflexion sur le projet en question (cf. résumé).

14. Les résumés de ces communications sont en ligne : https://www.college-de-france.fr/site/ vinciane-pirenne-delforge/symposium-2017-2018.htm. 


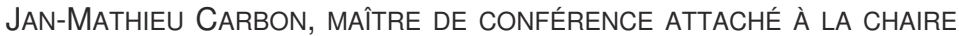

Outre sa contribution au projet CGRN, Jan-Mathieu Carbon a entrepris la rédaction d'une monographie sur les calendriers sacrificiels des îles voisines de Cos et de Rhodes ; étudiés et (dans le cas de Rhodes) édités pour la première fois de manière exhaustive, les calendriers de ces deux états insulaires servent ici de base à une plus large enquête sur ces communautés grecques et leurs univers panthéoniques ; l'enjeu consiste à comprendre comment se présentait l'année religieuse dans ces cités ; il participe aussi à la publication d'un corpus des inscriptions d'Halicarnasse, un projet mené en collaboration avec Signe Isager et Poul Pedersen, respectivement professeurs émérites des universités d'Odense et d'Aarhus.

Publications :

Carbon J.-M. et Peels-Matthey S. (dir.), Purity and Purification in the Ancient Greek World: Texts, Rituals, and Norms, Liège, Presses universitaires de Liège, coll. «Kernos Suppléments », vol. 32, 2018.

CARBON J.-M., "A network of hearths: Honours, sacrificial shares, and "traveling meat" », in J. BLOK, F. VAN DER EIJNDE et R. STROOTMAN (dir.), Feasting and Polis Institutions, Leyde/ Boston, Brill, coll. « Mnemosyne Supplements », vol. 414, 2018, p. 340-375.

CARBON J.-M., "Meaty perks: Epichoric and topological trends», in S. HITCH et I. RUTHERFORD (dir.), Animal Sacrifice in the Ancient Greek World, Cambridge, University Press of Cambridge, 2017, p. 151-177.

\section{PUBLICATIONS}

\section{OUVRAGE}

Pirenne-Delforge V., Le Polythéisme grec comme objet d'histoire, Paris, Collège de France/ Fayard, coll. «Leçons inaugurales du Collège de France », vol. 274, 2018 ; édition numérique : Collège de France, 2019, DOI : 10.4000/books.cdf.6159; en ligne : http://books.openedition. org/cdf/6159.

\section{ARTICLE}

PIRENNE-DeLForge V., «Greek gods and cognitive sciences: About Jennifer Larson's Understanding Greek religion », Journal of Cognitive Historiography, vol. 4, $\mathrm{n}^{\circ}$ 1, 2017, p. 47-52, DOI : 10.1558/jch.36262.

\section{CHAPITRES DE LIVRES}

PiRENNE-Delforge V., "Vernant, le sacrifice et la cuisine, quarante ans après... », in S. Georgoudi et F. DE Polignac (dir.), Relire Vernant, Paris, Les Belles Lettres, 2018, p. 53-81 [https://orbi.uliege.be/handle/2268/86802].

PIRENNE-DELForge V., « Nyx est, elle aussi, une divinité : La Nuit dans les mythes et les cultes grecs », in A. ChANIOTIS (dir.), La Nuit : imaginaire et réalités nocturnes dans le monde grécoromain. Neuf exposés suivis de discussions, Genève/Vandoeuvres, Droz, coll. «Entretiens sur l'Antiquité classique », vol. 64, 2018, p. 131-165, https://orbi.uliege.be/handle/2268/227498. 
SCHEID J. et PIRENNE-DELFORGE V., «Vernant, les dieux et les rites: héritages et controverses », in C. Bonnet, N. Belayche, M. Albert LlorCA, A. AfdeEFf, F. Massa et I. SlobodzianeK (dir.), Puissances divines à l'épreuve du comparatisme. Constructions, variations et réseaux relationnels, Turnhout, Brepols, coll. «Bibliothèque de l'École des Hautes Études. Sciences religieuses », vol. 175, 2017, p. 33-52, DOI : 10.1484/M. BEHE-EB.5.114074.

Pirenne-Delforge V. et Pironti G., « Vénus et Aphrodite : 60 ans après la thèse de Robert Schilling », in N. BELAYCHE et Y. LEHMANN (dir.), Religions de Rome. Dans le sillage des travaux de Robert Schilling, Brepols, coll. «Recherches sur les rhétoriques religieuses », vol. 21, 2017, p. 19-45, http://hdl.handle.net/2268/212709.

PIRENNE-Delforge V., «Pure and impure ancestors at Selinous: A note on Greek theology », in K. BielawsKi (dir.), Animal Sacrifice in Ancient Greece: Proceedings of the First International Workshop in Kraków (12-14.11.2015), Varsovie, 2017, p. 67-86, DOI : 10.1484/M.RRR-EB.5.113278, http://hdl.handle.net/2268/213315.

PIRONTI G. et PiRENNE-DELFORGE V., « Humnétriai, chœur de jeunes filles... : un rite mis en images chez Philostrate », in C. CALAME et P. Ellinger (dir.), Du récit au rituel par la forme esthétique. Poèmes, images et pragmatique cultuelle en Grèce ancienne, Paris, Les Belles Lettres, 2017, p. 241-268, http://hdl.handle.net/2268/161513.

Pirenne-Delforge V., «Héra, Apollon et l'Héraion de Délos à la période archaïque », in A. MAZARAKIS AINIAN (dir.), Les Sanctuaires archaïques des Cyclades, Presses universitaires de Rennes, coll. «Archéologie \& culture », 2017, p. 91-104, http://hdl.handle. net/2268/205539.

\section{AUTRE PUBLICATION}

Pirenne-Delforge V. et Pironti G., «Hera », Oxford Classical Dictionary, Oxford University Press, 2017, http://hdl.handle.net/2268/215461.

\section{Publications COMMUNeS DE L'ÉQUIPE}

CARbon J.-M. et PIRENNE-Delforge V., «Codifying "Sacred Laws" in Ancient Greece », in D. Jaillard et C. NihAN (dir.), Writing Laws in Antiquity / L'Écriture du droit dans l'Antiquité, Wiesbaden, Harrassowitz, coll. « Beihefte zur Zeitschrift für die Altorientalische und Biblische Rechtsgeschichte », vol. 19, 2017, p. 141-157, http://hdl.handle.net/2268/133370.

Pirenne-Delforge V., Carbon J.-M. et Peels S., Collection of Greek Ritual Norms, 2017, http://cgrn.ulg.ac.be (base de données, site internet). 
